PROCEEDINGS OF THE

AMERICAN MATHEMATICAL SOCIETY

Volume 126, Number 11, November 1998, Pages 3407-3411

S 0002-9939(98)04374-3

\title{
A COMPACT HL-SPACE NEED NOT HAVE A MONOLITHIC HYPERSPACE
}

\author{
HENNO BRANDSMA AND JAN VAN MILL
}

(Communicated by Alan Dow)

\begin{abstract}
We show that Kunen's example of a compact L-space, constructed under $\mathrm{CH}$, can be modified so that it has a non-monolithic hyperspace. This answers a question of Bell's. This example is also relevant to a question of Arhangel'skiî's.
\end{abstract}

\section{INTRODUCTION}

A topological space $X$ is called monolithic if for every subspace $A$ of $X$ we have $n w(\bar{A}) \leq|A|$, where $n w$ denotes the net weight of a space. As we are only interested in compact $X$, we will use the above definition with $n w(\bar{A})$ replaced by $w(\bar{A})$, where $w$ denotes the weight of a space. Arhangel'skil asked in [1, problem II.9]: "When is $H(X)$ monolithic?" Here $H(X)$ denotes the hyperspace of non-empty closed subsets of $X$ endowed with the Vietoris topology. Bell [2] answered this question in the case $X$ is orderable: For compact ordered $X$ the monolithicity of $H(X)$ is equivalent to $X$ being monolithic and hereditarily Lindelöf (HL). Note that by proposition 2.2 of Bell's paper all the conditions on $X$, including compactness, are already necessary for $H(X)$ to be monolithic. He also noted that under MA $\left(\omega_{1}\right)$ the monolithicity of $H(X)$ is equivalent to $X$ being a metrisable compactum, and that under $\neg \mathrm{SH}$ a Souslin line would be an example of a non-metrisable HL compactum with a monolithic hyperspace. Bell also asked ([2, Question 2.4]) whether Kunen's compact L-space, constructed under $\mathrm{CH}$, is another such example (see [4]). We will show that this need not be the case, by modifying Kunen's construction to ensure that its hyperspace is non-monolithic. This shows that the above necessary conditions are in general not sufficient for $H(X)$ to be monolithic. Note that Kunen's L-space is indeed monolithic, as every separable subspace has a countable base, and its weight is $\omega_{1}$. We were unable to solve the following:

Question 1. If there is a non-metrisable HL monolithic compactum with a monolithic hyperspace, does it follow that there exists a Souslin line?

Our example is obtained by making specific choices for certain subsets in Kunen's general construction procedure. So we may ask the following:

Question 2. Does every space constructed like Kunen's L-space have a non-monolithic hyperspace?

Received by the editors July 30, 1996 and, in revised form, March 19, 1997.

1991 Mathematics Subject Classification. Primary 54A20, 54B20, 54D30, 54G20.

Key words and phrases. Hyperspaces, monolithic, compact spaces, counterexamples.

(C)1998 American Mathematical Society 
Of course, a negative answer to this second question would give a negative answer to the first one, as the existence of a Souslin line is independent of $\mathrm{CH}$. However, we suspect the answer to the second question will be affirmative.

\section{The COnstruction}

We will first briefly review Kunen's construction from [4]. This construction will leave us with a lot of freedom, with which we will ensure the eventual nonmonolithicity of the hyperspace.

A similar construction can be found in [5], where inverse limits of spaces were used to construct non-metrisable hereditarily separable spaces with special properties. There also measures play an important role in the arguments. In Banach space theory similar examples were constructed by Haydon and Talagrand. In [6] the reader can find an alternative construction of Kunen's L-space that also has the Banach space properties that Haydon and Talagrand were after.

The space $X$ that we're after will be constructed as the limit of an inverse sequence of metrisable compacta of length $\omega_{1}$. This sequence of spaces will be denoted by $X_{\alpha}$, where $\omega \leq \alpha \leq \omega_{1}$. As in [4], each $X_{\alpha}$ will be constructed as a closed subspace of $2^{\alpha}$ endowed with the usual product topology. At limit stages $\gamma, X_{\gamma}$ will be the inverse limit of the previous $X_{\alpha}$, which can be identified with a subspace of $2^{\gamma}$ in a canonical way: $X_{\gamma}$ is just the set of all points of $2^{\gamma}$ such that all projections onto previous stages $\alpha$ are in $X_{\alpha}$. All the projections of the inverse limit will be restrictions of the canonical projections between the different powers of 2. Moreover, each of these spaces will carry a Baire probability measure $\mu_{\alpha}$ which behaves well w.r.t. the inverse system projections. That is, we will require of our construction the following:

$$
\text { If } \alpha \leq \beta \text {, then } X_{\alpha}=\pi_{\alpha}^{\beta}\left(X_{\beta}\right)
$$

and

$$
\mu_{\alpha}(Y)=\mu_{\beta}\left(\left(\pi_{\alpha}^{\beta}\right)^{-1}(Y)\right) \text { for any Baire subset } Y \text { of } X_{\alpha} .
$$

Furthermore we will need the following two "easy" conditions on our construction:

$$
\text { For each } \alpha, \mu_{\alpha}(\{p\})=0 \text { for all } p \in X_{\alpha}
$$

and

$$
\text { For each } \alpha, \mu_{\alpha}(K)>0 \text { for all non-empty clopen } K \subset X_{\alpha} \text {. }
$$

Here $\pi_{\alpha}^{\beta}$ denotes the natural projection from $2^{\beta}$ onto $2^{\alpha}$. We will also use the notation $p_{\alpha}^{\beta}$ for the restriction of this map to $X_{\beta}$ and $X_{\alpha}$. Note that $X_{\gamma}$, where $\gamma$ is a limit ordinal, is completely determined by its predecessors: it's just the measuretheoretic (and topological) inverse limit of it, and it automatically satisfies (3) and (4) if its predecessors do. Also, there is no problem starting the construction: just take $X_{\omega}$ to be $2^{\omega}$ with its standard product measure. Kunen's last two conditions are the really essential ones: (5) is needed to give closed sets of positive measure an interior. This is needed to let every nowhere dense set in $X$ have measure 0 . (6) is used to show that all nowhere dense sets in $X$ have a countable base. These two properties of the nowhere dense subsets of $X$ are basically responsible for making $X$ HL but not separable.

At the beginning of the construction we fix, using $\mathrm{CH}$, for each $\alpha$ such that $\omega \leq \alpha<\omega_{1}$ an enumeration $\left\{F_{\xi}^{\alpha}: \xi<\omega_{1}\right\}$ of the non-empty closed subsets of 
$2^{\alpha}$. Also we fix a function $g$ from $\omega_{1}$ onto $\omega_{1} \times \omega_{1}$ such that $g(\beta)=(\alpha, \xi)$ implies $\alpha \leq \beta$. Now we can formulate the last two conditions:

(5) For each $\beta<\omega_{1}$ : If $g(\beta)=(\alpha, \xi), F_{\xi}^{\alpha} \subset X_{\alpha}$, and $\mu_{\alpha}\left(F_{\xi}^{\alpha}\right)>0$,

then there is a closed $C \subset\left(\pi_{\alpha}^{\beta}\right)^{-1}\left(F_{\xi}^{\alpha}\right)$ such that

$$
\mu_{\beta}(C)>0 \text { and } X_{\beta+1}=X_{\beta} \times\{0\} \cup C \times\{1\} .
$$

(6) For each $\beta<\omega_{1}$ and all $\alpha, \xi \leq \beta$ : If

$$
\begin{aligned}
& F_{\xi}^{\alpha} \subset X_{\alpha} \text { and } \mu_{\alpha}\left(F_{\xi}^{\alpha}\right)=0, \\
& \text { then } X_{\beta+1} \cap\left(\left(\pi_{\alpha}^{\beta}\right)^{-1}\left(F_{\xi}^{\alpha}\right) \times\{1\}\right)=\emptyset .
\end{aligned}
$$

These two conditions are the ones we have to preserve at successor stages in order to get Kunen's L-space. However, we will need to build in some extra features to ensure that its hyperspace will be non-monolithic. To begin with, let $\mathcal{B}$ be the collection of clopen sets of $X_{\omega}=2^{\omega}$. We want to make sure that the (countable) collection $\left\{\left(p_{\omega}^{\omega_{1}}\right)^{-1}(B): B \in \mathcal{B}\right\}$ will be a set in the hyperspace of $X_{\omega_{1}}$ with non-metrisable closure.

To do this we will need to make the projection from $H\left(X_{\beta+1}\right)$ to $H\left(X_{\beta}\right)$ more-toone on the closure of the set $\left\{\left(p_{\omega}^{\beta+1}\right)^{-1}(B): B \in \mathcal{B}\right\}$, at least for cofinally many $\beta$. This is the extra requirement needed in the construction of $X$. (Later on we will see why this is enough.) Let us first show how to meet requirements (1) to (6) on successor steps: this is just following Kunen's original construction. So assume we are at stage $\beta$ in our inductive construction and that all previously constructed $X_{\alpha}$ already satisfy these requirements. Let $g(\beta)=(\alpha, \xi)$. If it is not the case that both $F_{\xi}^{\alpha} \subset X_{\alpha}$ and $\mu_{\alpha}\left(F_{\xi}^{\alpha}\right)>0$, then conditions (5) and (6) are empty and we can fulfill everything by chosing $X_{\beta+1}=X_{\beta} \times\{0\}$ with the obvious measure. We don't care about making the hyperspace map more-to-one in this case.

Otherwise, let

$$
D=\left(\pi_{\alpha}^{\beta}\right)^{-1}\left(F_{\xi}^{\alpha}\right) \backslash \bigcup\left\{\left(\pi_{\delta}^{\beta}\right)^{-1}\left(F_{\eta}^{\delta}\right): \delta, \eta \leq \beta, F_{\eta}^{\delta} \subset X_{\delta}, \mu_{\delta}\left(F_{\eta}^{\delta}\right)=0\right\} .
$$

Then $D$ is a Baire set of positive measure, so we can choose a closed $E \subset D$ with positive measure (by regularity of $\mu_{\beta}$ ). Now let

$$
C=E \backslash \bigcup\left\{K \subset X_{\beta}: K \text { is clopen and } \mu_{\beta}(E \cap K)=0\right\} .
$$

$\mathrm{C}$ is a closed set with the same measure as $E$, which moreover has the following property

$$
\forall K \subset X_{\beta}:\left(K \text { clopen and } \mu_{\beta}(C \cap K)=0\right) \Rightarrow C \cap K=\emptyset .
$$

If we now "split" any positive measure closed subset of $C$, which also has the property $(*)$ above, we will satisfy all the conditions (1) to (6) above. Kunen just split the above $C$ to get his L-space. We will need to do more work. We will construct a closed set $F$ in $X_{\beta}$ and a disjoint sequence $B_{n}$ of elements of $\mathcal{B}$ such that the following will hold:

(i) For all $n,\left(p_{\omega}^{\beta}\right)^{-1}\left(B_{n}\right) \cap F=\emptyset$.

(ii) $\left(p_{\omega}^{\beta}\right)^{-1}\left(B_{n}\right)$ converges to a subset $F^{\prime}$ of $F$ in the hyperspace of $X_{\beta}$.

(iii) $\sum_{n \in \omega} \mu_{\omega}\left(B_{n}\right)<\frac{1}{2} \mu_{\beta}(C)$.

(iv) For all $n, \mu_{\beta}\left(\left(p_{\omega}^{\beta}\right)^{-1}\left(B_{n}\right) \cap C\right)>0$. 
To do this, first note that the measure of the compact set $C^{\prime}=p_{\omega}^{\beta}(C)$ cannot be 0 , because $C$ has positive measure. So in $C^{\prime}$ there will be a sequence $y_{n}$ converging to a point $y$ of $C^{\prime}$. Let the $K_{n}$ be clopen neighbourhoods of $y_{n}$ missing the point $y$ such that the $K_{n}$ 's are pairwise disjoint, and such that they satisfy (iii). We also want the diameter of $K_{n}$ to tend to 0 as $n$ goes to infinity. It is obvious that this can be done. Now consider the (disjoint) sequence $\left(p_{\omega}^{\beta}\right)^{-1}\left(K_{n}\right)$ in the hyperspace of $X_{\beta}$. By compactness of this hyperspace, there will be a subsequence of it that converges to some closed set $F^{\prime} \subset X_{\beta}$. Taking the corresponding $K_{n}$ 's will give the required sequence $B_{n}$. The set $F$ will be $\left(p_{\omega}^{\beta}\right)^{-1}(y)$. By sequential continuity of the map $p_{\omega}^{\beta}$ (as a map between the hyperspaces) and the fact that the $K_{n}$ 's converge to $\{y\}$, we see that $F^{\prime} \subset F$, as required. It is obvious that all the requirements on $B_{n}$ are met; (iv) follows because of the property (*) for $C$. Let us reenumerate the odd terms of the sequence $\left(p_{\omega}^{\beta}\right)^{-1}\left(B_{n}\right)$ as $A_{n}$ and the even terms as $C_{n}$. We now define $S$ as

$$
C \backslash \bigcup\left\{A_{n}: n \in \omega\right\}
$$

By removing, as before, the relatively clopen subsets of $\mathrm{S}$ of measure 0 we will have an $S^{\prime}$ which has property $*$. This is the set we will split. Observe that it has positive measure, because what we remove above has at most measure $\frac{1}{2} \mu_{\beta}(C)$ by requirement (iii), and of course it is still closed. Also, it is still the case that all $C_{n}$ intersect $S^{\prime}$, because we remove sets of measure 0 from $\mathrm{S}$, with which the intersection has positive measure by (iv). Now we can define $X_{\beta+1}$ to be $\left(X_{\beta} \times\{0\}\right) \cup\left(S^{\prime} \times\{1\}\right)$ and $\mu_{\beta+1}$ will be given by

$$
\mu_{\beta+1}(A \times\{0\})=\mu_{\beta+1}(A \times\{1\})=\frac{1}{2} \mu_{\beta}(A) \text { for } A \subset S^{\prime},
$$

and $\mu_{\beta+1}(A \times\{0\})=\mu_{\beta}(A)$ for $A \subset X_{\beta} \backslash S^{\prime}$. It is easy to check that this $X_{\beta+1}$ and $\mu_{\beta+1}$ fulfill all the requirements; that it satisfies (4) is checked using property $(*)$ for $S^{\prime}$.

We will now show that the projection $p=p_{\beta}^{\beta+1}$, considered as a map between the hyperspaces, is not one-to-one on the closure of $\left\{\left(p_{\omega}^{\beta+1}\right)^{-1}(B): B \in \mathcal{B}\right\}$. On the one hand, because the $A_{n}$ 's are disjoint from $S^{\prime}$ in $X_{\beta}$, we have that $p^{-1}\left(A_{n}\right) \subset X_{\beta} \times\{0\}$ in $H\left(X_{\beta+1}\right)$. and this sequence converges to $F^{\prime} \times\{0\}$ in this hyperspace. On the other hand, we have that $p^{-1}\left(C_{n}\right) \cap\left(X_{\beta} \times\{0\}\right)$ converges to $F^{\prime} \times\{0\}$ in the hyperspace of $X_{\beta} \times\{0\}$ and that a subsequence of $p^{-1}\left(C_{n}\right) \cap\left(S^{\prime} \times\{1\}\right)$ converges to some subset of the form $F^{\prime \prime} \times\{1\}$, where $F^{\prime \prime}$ is a closed subset of $F^{\prime}$ (by compactness of $H\left(S^{\prime} \times\{1\}\right.$ ). It is obvious that both $p^{-1}\left(A_{n}\right)$ and $p^{-1}\left(C_{n}\right)$ are elements of the set $\left\{\left(p_{\omega}^{\beta+1}\right)^{-1}(B): B \in \mathcal{B}\right\}$, so it now follows that $F^{\prime} \times\{0\}$ and $\left(F^{\prime} \times\{0\}\right) \cup\left(F^{\prime \prime} \times\{1\}\right)$ are both elements of the closure of this set. Now, $p$ maps both these elements of $H\left(X_{\beta+1}\right)$ to the same element $F^{\prime}$ of $H\left(X_{\beta}\right)$, so $p$ is not one-to-one on the closure of $\left\{\left(p_{\omega}^{\beta+1}\right)^{-1}(B): B \in \mathcal{B}\right\}$. Also note that this happens cofinally many times, because we perform the above step cofinally many times as well (if not, the resulting space would be metrisable, which it obviously is not).

Now let $X$ be $X_{\omega_{1}}$ and let $\mu$ be $\mu_{\omega_{1}}$. Using the same proof as in [4], one can show that $X$ is an HL space, which is not separable, and that for every subset $Y$ of $X$ the following are equivalent:

(a) $\mu(Y)=0$.

(b) $Y$ is first category in $X$. 
(c) $Y$ is nowhere dense in $X$.

(d) $Y$ is separable.

(e) $Y$ is second countable.

That (d) implies (e), combined with the fact that the weight of $X$ is $\omega_{1}$, implies that $X$ is monolithic.

Now we will see that we have made $H(X)$ non-monolithic. Let us introduce some notation first. We will use the notation $\hat{p}_{\xi}^{\alpha}$ for the map $p_{\xi}^{\alpha}$ viewed as a map between $H\left(X_{\alpha}\right)$ and $H\left(X_{\xi}\right)$ in the obvious way. It is well known that with these maps as bonding maps we get an inverse system of hyperspaces which has the hyperspace of $X$ as its limit (cf. [3, 3.12.27(f)]). Another well known fact is that if we have a closed metrisable subset $K$ in this limit, the canonical projection from the limit restricted to $K$ will be one-to-one from a certain index onward. (This can be seen using the compactness of all spaces involved combined with a countable base of this subspace. Alternatively, we could apply $[3,2.5 .6]$ to see that $K$ itself is an inverse limit of closed subsets of $H\left(X_{\alpha}\right)$, and then apply [3, 3.2H(e)] to the identity mapping of $K$.) Now, let $\mathcal{B}_{\alpha}$ be the countable collection $\left\{\left(p_{\omega}^{\alpha}\right)^{-1}(B): B \in \mathcal{B}\right\}$ in $H\left(X_{\alpha}\right)$, for all $\omega \leq \alpha \leq \omega_{1}$. Then we obviously have that $\hat{p}_{\xi}^{\alpha}$ maps $\mathcal{B}_{\alpha}$ onto $\mathcal{B}_{\xi}$ for all $\xi \leq \alpha \leq \omega_{1}$ and that the analogous statement for the closures of these sets in the hyperspace is also true by the closedness of all maps involved. Assume that $\overline{\mathcal{B}}_{\omega_{1}}$ is metrisable. By the above remark this would imply that there is a certain index $\alpha$ such that for all $\beta \geq \alpha$ we have that $\hat{p}_{\beta}^{\omega_{1}}$ is one-to-one on $\overline{\mathcal{B}}_{\omega_{1}}$. This would

then imply that for such $\beta$ we have that $\hat{p}_{\beta}^{\beta+1}$ is one-to-one on $\overline{\mathcal{B}}_{\beta+1}$. But we have seen above that this is not the case for cofinally many $\beta$. This contradiction proves that the closure of $\left\{\left(p_{\omega}^{\omega_{1}}\right)^{-1}(B): B \in \mathcal{B}\right\}$ is not metrisable, and hence that $H(X)$ is not monolithic.

\section{Note Added in Proof (February 12, 1998)}

The authors recently proved the answer to Question 2 to be affirmative (preprint Sept. 1997).

\section{REFERENCES}

1. A.V. Arhangel'skiı̌, Topological Homogeneity. Topological Groups and their Continuous Images, Russian Math. Surveys 42 (1987), no. 2, 83-131. MR 89b:54004

2. M. Bell, The Hyperspace of a Compact Space I, Topology and its Applications 72 (1996), 39-46. MR 97j:54010

3. R. Engelking, General Topology, revised and completed ed., Sigma series in pure mathematics, vol. 6, Heldermann Verlag Berlin, 1989. MR 91c:54001

4. K. Kunen, A Compact L-space under CH, Topology and its Applications 12 (1981), 283-287. MR 82h: 54065

5. K. Kunen and M. Džamonja, Measures on Compact HS spaces, Fundamenta Mathematicae 143 (1993), 41-54. MR 94h:54047

6. S. Negrepontis, Banach Spaces and Topology, Handbook of Set-Theoretic Topology (K. Kunen and J.E. Vaughan, eds.), North-Holland, 1984. MR 86i:46018

Vrije Universiteit, Faculty of Mathematics and Computer Science, De Boelelaan 1081A, 1081 HV Amsterdam, The Netherlands

E-mail address: hsbrand@cs.vu.nl

E-mail address: vanmill@cs.vu.nl 